\title{
Lysosomal acid lipase activity and liver fibrosis in the clinical continuum of non-alcoholic fatty liver disease
}

\author{
Francesco Baratta ${ }^{1,2 *}$ | Daniele Pastori ${ }^{1 *}$ (D) | Giulia Tozzi ${ }^{3}$ | Laura D'Erasmo ${ }^{1}$ | \\ Alessia Di Costanzo $^{1}$ | Marcello Arca ${ }^{1}$ | Evaristo Ettorre ${ }^{4}$ | Stefano Ginanni Corradini ${ }^{5}$ | \\ Francesco Violi $^{1,6}$ (i) | Francesco Angelico ${ }^{7}$ (D) | Maria Del Ben ${ }^{1}$
}

${ }^{1}$ I Clinica Medica, Department of Internal Medicine and Medical Specialties, Sapienza University of Rome, Rome, Italy

${ }^{2}$ Department of Anatomical Sciences, Histological, Legal Medical and

Locomotor, Sapienza University of Rome, Rome, Italy

${ }^{3}$ Hepatology, Gastroenterology and Nutrition Unit, IRCCS "Bambino Gesù" Children's Hospital, Rome, Italy

${ }^{4}$ Division of Gerontology, Department of Cardiovascular, Respiratory,

Nephrologic, Anesthesiologic and Geriatric

Sciences, Sapienza University, Rome, Italy

${ }^{5}$ Department of Translational and Precision Medicine, Sapienza University of Rome, Rome, Italy

${ }^{6}$ Mediterranea Cardiocentro, Naples, Italy

${ }^{7}$ Department of Public Health and Infectious Diseases, Sapienza University of Rome,

Rome, Italy

Correspondence

Dr Daniele Pastori, I Clinica Medica, Department of Internal Medicine and Medical Specialties, Sapienza University of Rome, Rome Italy.

Email: daniele.pastori@uniroma1.it

Handling Editor: Luca Valenti

\begin{abstract}
Background and Aims: Recent evidence showed a reduced activity of the lysosomal acid lipase (LAL) in patients with non-alcoholic fatty liver disease (NAFLD) and cryptogenic cirrhosis (CC). However, the relationship between LAL activity and liver fibrosis has never been investigated.

Methods: Cross-sectional study including 575 outpatients referred for the management of cardio-metabolic and liver disease. The absence of liver fibrosis was defined by a FIB-4 < 1.30 and NAFLD fibrosis score (NFS) $<-1.455$. LAL activity was measured with dried blood spot technique.

Results: Overall, 515 patients had a diagnosis of NAFLD (454 NAFL and 61 biopsyproven NASH) and 60 of CC. The value of LAL activity progressively decreased from healthy subjects to NAFL/NASH patients to CC $(P<.001)$. LAL activity was reduced by $10 \%$ in patients with NAFL, by $20 \%$ in NASH and by $50 \%$ in CC. The prevalence of CC decreased across the tertiles of LAL activity: $22.2 \%$ in the lowest, $4.6 \%$ in the intermediate and $0.5 \%$ in the highest tertile. In NAFLD patients, $69.9 \%$ had a FIB4 < 1.30, and 43.1\% a NFS <-1.455. Multivariate logistic regression analysis showed that Log (LAL activity) was associated with FIB-4 $<1.30$ (Odds ratio [OR] $2.1995 \%$ confidence interval $[\mathrm{Cl}]$ 1.33-3.62, $P=.002$ ) and NFS < -1.455 (OR 2.43, $95 \% \mathrm{Cl} 1.51-3.91, P<.001)$ after adjustment for confounding factors.

Conclusions: We found a progressive reduction of LAL activity according to liver disease severity. LAL activity was inversely associated with markers of liver fibrosis in patients with NAFLD.
\end{abstract}

\section{KEYWORDS}

cryptogenic cirrhosis, liver fibrosis, lysosomal acid lipase, NAFLD

\footnotetext{
Abbreviations: ALT, alanine aminotransferase; AST, aspartate aminotransferase; ATP, adult treatment panel; CC, cryptogenic cirrhosis; CESD, cholesterol ester storage disease; CPT, Child-Pugh-Turcotte; DBS, dried blood spot; FIB-4, fibrosis-4; GGT, $\gamma$-glutamyltransferase; HDL, high-density lipoprotein; HS, healthy subjects; LAL, lysosomal acid lipase; LAL-D, lysosomal acid lipase deficiency; LIPA, lipase a, lysosomal acid; NAFLD, non-alcoholic fatty liver disease; NAS, NAFLD activity score; NASH, non-alcoholic steatohepatitis; NFS, NAFLD fibrosis score; OR, odds ratio; PNPLA3, patatin-like phospholipase domain-containing protein 3.
}

*Equal contribution 


\section{1 | INTRODUCTION}

Non-alcoholic fatty liver disease (NAFLD) includes a broad spectrum of liver disorders, which may progress from simple steatosis (NAFL) to non-alcoholic steatohepatitis (NASH), NASH cirrhosis, hepatocellular carcinoma and liver-related death.

Non-alcoholic fatty liver disease represents the most common and emerging chronic liver disease worldwide with an estimated prevalence of $25 \%-30 \%$ in the general population rising to $50 \%$ in some clinical conditions such as diabetes, metabolic syndrome and obesity. Most patients develop a mild disease with minimal inflammation and no liver fibrosis, and about $25 \%$ of patients affected by NASH may develop significant fibrosis and eventually cirrhosis.

The pathogenesis of NAFLD appears to be multifactorial and many mechanisms promoting the excessive fat accumulation in the liver have been proposed, including patatin-like phospholipase domain-containing protein 3 (PNPLA3) genetic mutation and insulin resistance. ${ }^{1,2}$ However, the pathogenic mechanisms that promote the development of significant liver inflammation and fibrosis are not yet fully elucidated. This is of concern as the presence of liver fibrosis has been shown to be the most important factor worsening the prognosis of patients with NAFLD. ${ }^{3}$

Lysosomal acid lipase ( $L A L)$ is a hydrolase that plays a key role in intracellular cholesterol trafficking. A reduced LAL (LAL-D) activity promotes increased lysosomal cholesterol ester storage, as observed in two recessive autosomal genetic diseases, namely Wolman disease and Cholesterol ester storage disease (CESD). LAL activity is absent in Wolman disease and greatly reduced $(<10 \%)$ in the case of the CESD. The most common mutation is the E8SJM variant, a single mutation affecting the LIPA gene. However, more than 40 loss-offunction mutations have been reported and, at the moment, no data are present on the modulation of LAL activity in vivo, nor on the epigenetic and metabolic factors able to regulate its activity in subjects without mutations of the LIPA gene.

Previous studies showed a progressive decrease in LAL activity from healthy subjects (HS) to subjects with NAFL and those with biopsy-proven NASH. ${ }^{4,5}$ More recently, in patients with cryptogenic cirrhosis (CC), a further reduction in the activity of LAL has been described. ${ }^{6,7}$ These results were similar to those obtained in children with biopsy-proven NASH. ${ }^{8}$

However, the relationship between LAL activity and liver fibrosis has not been investigated in adult patients with NAFLD.

The aims of the study were to evaluate $L A L$ activity across the wide clinical continuum of NAFLD and to assess the relationship between LAL activity and surrogate markers of liver fibrosis in this clinical setting.

\section{2 | MATERIALS AND METHODS}

We performed a cross-sectional study which included 583 consecutive outpatients referring to the Day Service of Internal Medicine and Metabolic Diseases of the Department of Internal Medicine of

\section{Key points}

- Lysosomal acid lipase (LAL) activity progressively reduces with liver disease severity by $10 \%$ in patients with NAFL, by $20 \%$ in NASH and by $50 \%$ in cryptogenic cirrhosis (CC).

- In patients with non-alcoholic fatty liver disease (NAFLD), LAL activity is inversely associated with noninvasive markers of liver fibrosis.

Sapienza University of Rome for the management of cardio-metabolic risk factors, such as metabolic syndrome, dyslipidemia, arterial hypertension, diabetes and liver diseases. Inclusion criteria for the study were: no history of alcohol consumption above $20 \mathrm{gr} /$ daily; no evidence of hepatitis C-B virus infections or autoimmune or druginduced hepatitis. In addition, the study also included a cohort of 60 patients with cryptogenic cirrhosis (CC) followed up at the liver disease outpatient clinic.

Lysosomal acid lipase activity values from 100 voluntary HS, defined as subjects without evidence of any diseases, not taking drugs and without fatty liver at liver ultrasonography scanning (US) are reported in Figure 1 as reference of normal value. The reduction in LAL activity was defined by a value below the 2.5 th percentile $(<0.8 \mathrm{nmol} / \mathrm{spot} / \mathrm{h})$ of LAL activity from $\mathrm{HS}$, as previously described. ${ }^{4}$

At first visit, a liver US was performed and data on comorbidities and previous major adverse cardiovascular and cerebrovascular events (MACCE) were collected. Metabolic syndrome was diagnosed according to the ATP III-modified criteria. ${ }^{9}$ The presence of diabetes and arterial hypertension was defined according to international guidelines. ${ }^{10,11}$

Moreover, patients underwent a complete clinical and biochemical diagnostic work-up including serum alanine aminotransferase (ALT), aspartate aminotransferase (AST), $\gamma$-glutamyltransferase (GGT), fasting total and HDL cholesterol, triglycerides, glucose, creatinine, albumin and a complete blood count.

\section{1 | Definition of liver diseases}

Liver steatosis was defined by ultrasonography (US) according to Hamaguchi criteria based on the presence of abnormally intense, high level echoes arising from the hepatic parenchyma, liver-kidney difference in echo amplitude, echo penetration into deep portion of the liver and clarity of liver blood vessel structure. ${ }^{12}$ To include patients in the NAFLD group, the following were considered as exclusion criteria: average daily consumption of alcohol $>20 \mathrm{~g}$ in women and $>30 \mathrm{~g}$ in men (assessed by Alcohol Use Disorders Identification Test, AUDIT ${ }^{13}$; the presence of hepatitis $B$ surface antigen and antibody to hepatitis $C$ virus; positive tests for autoimmune hepatitis; cirrhosis and other chronic liver diseases; diagnosis of oncological diseases and concomitant therapy with 


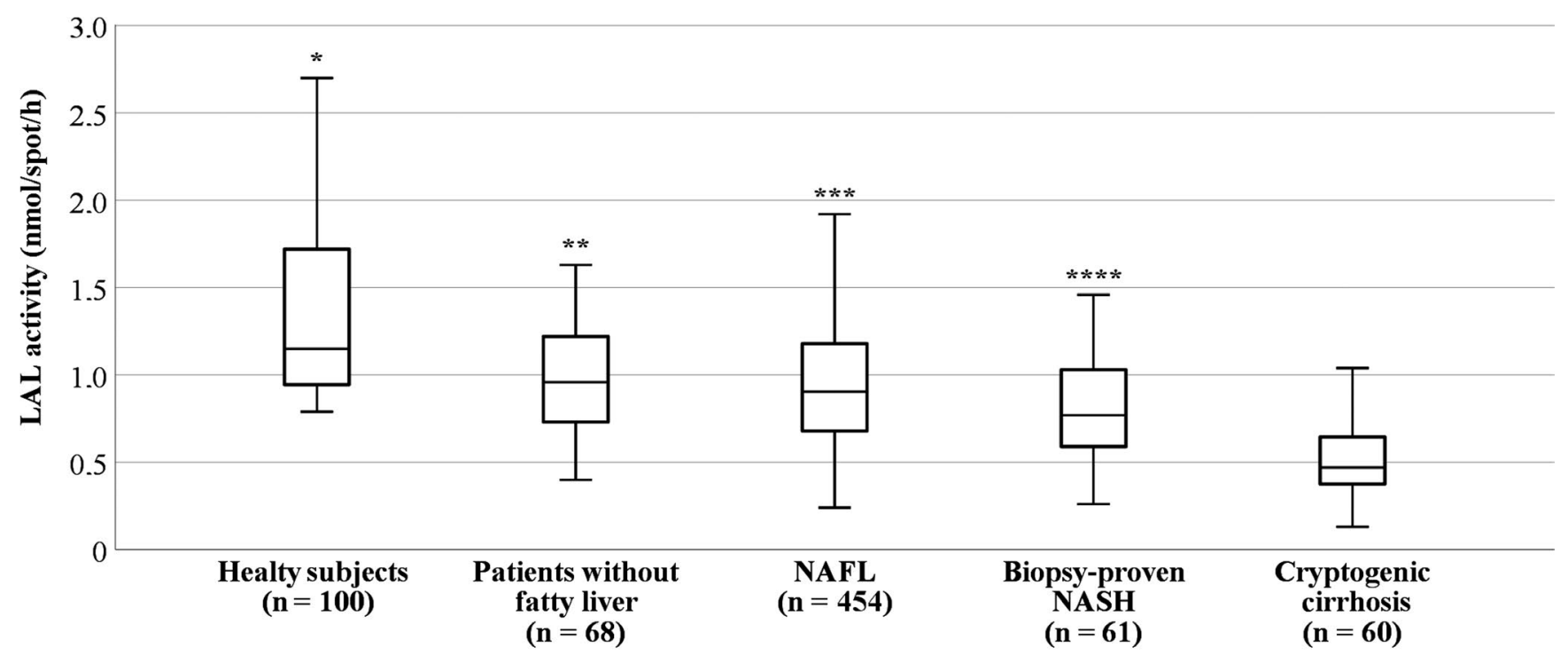

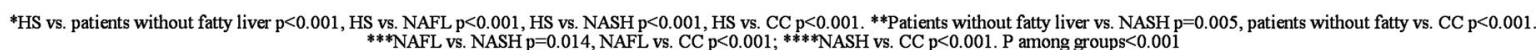

FIGURE 1 Lysosomal acid lipase (LAL) activity in the five study groups

drugs known to promote liver steatosis (eg amiodarone); other chronic infectious or autoimmune diseases; clinical, biochemical or US signs of cirrhosis or portal hypertension (nodular liver, ascites, portal flow mean velocity $<14 \mathrm{~cm} / \mathrm{s}$, inversion of flow in the portal vein, portosystemic collaterals, portal vein diameter $>13 \mathrm{~mm}$, decreased or no respiratory variation in splenic and superior mesenteric vein diameter, portal/splenic/superior mesenteric vein thrombosis). ${ }^{14,15}$

Percutaneous ultrasonography-guided liver biopsy was performed in NAFLD patients with clinical suspicion of NASH and persistent elevation of liver enzymes (>6 months). NASH was diagnosed in 61 patients by means of standard criteria based on separate scores for steatosis, hepatocellular ballooning and inflammation. ${ }^{16,17}$

Cryptogenic cirrhosis was defined as cirrhosis of unknown aetiology in patients with clinical history of previous overweight/obesity, diabetes, insulin resistance and/or liver steatosis and no history of alcoholism or alcohol consumption higher than $20 \mathrm{~g} / \mathrm{d}$ in men and $10 \mathrm{~g} / \mathrm{d}$ in women, or previous acute or chronic viral and autoimmune hepatitis. ${ }^{18}$ The severity of $\mathrm{CC}$ was assessed by the Child-PughTurcotte (CPT) score.

\section{2 | Non-invasive markers of liver fibrosis}

Fibrosis-4 (FIB-4) index and the NAFLD fibrosis score (NFS) are two simple non-invasive, accurate and validated methods to identify a high probability of severe fibrosis in adult patients with NAFLD. ${ }^{19,20}$ The use of these scores can accurately rule out the presence of liver fibrosis thus reducing the need for liver biopsy in most of the patients with NAFLD. Furthermore, these indexes can be easily calculated in large populations and repeated over time. Furthermore, high FIB-4 and NFS scores showed a prognostic value for liver disease mortality. ${ }^{21}$

\section{3 | LAL activity assessment and LIPA gene sequencing}

LAL activity was dosed with dried blood spot (DBS) technique using the inhibitor Lalistat 2 as previously reported, ${ }^{6}$ and expressed as $\mathrm{nmol} / \mathrm{spot} / \mathrm{h}$. DBS tests were performed in Bambino Gesù Hospital in Rome. Physicians analysing LAL activity were unaware of clinical and biochemical characteristics of any enrolled patient. Inter- and intra-assay variations were $2.4 \%$ and $2.3 \%$ respectively.

The LIPA gene was sequenced by the next-generation sequencing method as previously reported. ${ }^{22}$ In addition, to definitely exclude the presence of CESD-causing mutations, the Sanger method was used to resequence the Exon 8 of LIPA gene in NAFLD patients with a LAL activity value of $\leq 0.6 \mathrm{mmol} / \mathrm{spot} / \mathrm{h}$. Standard PCR and Sanger sequencing were performed as reported elsewhere. ${ }^{23}$ No CESD-causing mutations were found in all cases (data not shown).

Informed written consent was obtained, and the study protocol conformed to the ethical guidelines of the Declaration of Helsinki as reflected in a priori approval by the local ethical board of Sapienza University of Rome (ref. $n^{\circ} 2277 / 2011$ ).

\subsection{Statistical analysis}

Continuous variables were expressed as mean and standard deviation or median and interquartile range (IQR) depending on their distribution. Group comparisons were performed by unpaired Student's $t$ test and ANOVA, or by Mann-Whitney or Kruskal-Wallis test when appropriate. Proportions and categorical variables were tested by the $\chi^{2}$ test. Correlation analysis was performed by Spearman rank correlation test (rS).

We performed a descriptive analysis of characteristics of patients according to liver disease groups: Patients without fatty liver 
$(n=68) ; \operatorname{NAFL}(n=454) ; \mathrm{NASH}(\mathrm{n}=61) ; \mathrm{CC}(\mathrm{n}=60)$. A univariate and multivariate logistic regression analysis of factors associated with the clinical diagnosis of CC was performed to estimate the adjusted odds ratio (OR) with $95 \%$ confidence interval $(95 \% \mathrm{Cl}$ ) of each variable. In the multivariate model, variables presenting significant differences among groups, as reported in Table 1, were used as covariates. Then, factors associated with FIB-4 $<1.30$ and NFS $<-1.455$ were investigated by a multivariable logistic regression analysis.

For the logistic regression analysis, variables with non-normal distribution were log-transformed (ie LAL and AST values) or categorized (ie platelet count and albuminemia) when possible.

Variables were categorized or dichotomized for the logistic regression analysis. All tests were two-tailed, a $P$ value $<.05$ was considered statistically significant. Analyses were performed using computer software (sPss 23).

\section{3 | RESULTS}

In the entire cohort, 515 patients had a diagnosis of NAFLD (454 had NAFL and 61 biopsy-proven NASH), 68 were without steatosis at liver US and 60 had CC. Values of clinical and biochemical parameters in subjects belonging to the four groups are reported in Table 1.

\subsection{Characteristics of patients with CC}

In cirrhotic patients, $57.9 \%$ were CPT A, 35.1\% were CTP B and 7.0\% were CTP C. Patients with CC were older, more frequently diabetic and hypertensive and less frequently on statin therapy (Table 1). They presented worse liver function indexes, as shown by a lower mean albumin, cholesterol (total, HDL and LDL) and triglycerides, higher median liver function tests and lower platelet count (Table 1).

The value of LAL activity progressively decreased from HS to NAFL/NASH and CC (Table 1). In particular, a 10\% reduction in LAL activity was present in patients with NAFL, while those with NASH had a $20 \%$ reduction and those with CC had a $50 \%$ reduction (Figure 1). Moreover, the proportion of $\mathrm{LAL}<0.8 \mathrm{nmol} / \mathrm{spot} / \mathrm{h}$ increased from NAFL to NASH and CC (Figure 2).

In particular, the prevalence of $\mathrm{CC}$ decreased across the tertiles of LAL activity, with a higher prevalence in the lowest tertile (22.2\%)

TAB LE 1 Clinical and biochemical characteristics in patients without steatosis and in those with NAFL, NASH and cryptogenic cirrhosis (CC)

\begin{tabular}{|c|c|c|c|c|c|c|}
\hline & $\begin{array}{l}\text { Patients without } \\
\text { fatty liver }(n=68)\end{array}$ & NAFL ( $n=454)$ & NASH $(n=61)$ & $\begin{array}{l}\text { Cryptogenic cir- } \\
\text { rhosis }(n=60)\end{array}$ & $\begin{array}{l}P \text { (among } \\
\text { groups) }\end{array}$ & $\begin{array}{l}P(C C \text { vs } \\
\text { NAFLD) }\end{array}$ \\
\hline Age (y) & $59.3 \pm 13.9$ & $57.4 \pm 11.3$ & $49.5 \pm 12.9$ & $68.6 \pm 10.6$ & $<.001^{a}$ & $<.001$ \\
\hline Women (\%) & 50.0 & 39.6 & 37.7 & 28.3 & $.096^{b}$ & .122 \\
\hline Diabetes (\%) & 9.0 & 30.1 & 29.5 & 58.3 & $<.001^{\mathrm{b}}$ & $<.001$ \\
\hline Arterial hypertension (\%) & 58.5 & 74.5 & 55.9 & 50.0 & $<.001^{\mathrm{b}}$ & .001 \\
\hline Previous MACCE (\%) & 7.5 & 6.0 & 1.6 & 16.1 & .013 & .007 \\
\hline Blood glucose (mg/dL) & $95.2 \pm 19.2$ & $105.9 \pm 26.6$ & $100.0 \pm 18.0$ & $118.9 \pm 40.5$ & $<.001^{a}$ & .018 \\
\hline Total cholesterol (mg/dL) & $194.6 \pm 41.0$ & $197.9 \pm 39.8$ & $190.7 \pm 38.0$ & $153.1 \pm 41.7$ & $<.001^{a}$ & $<.001$ \\
\hline $\mathrm{HDL}(\mathrm{mg} / \mathrm{dL})$ & $58.2 \pm 12.2$ & $48.3 \pm 12.9$ & $49.4 \pm 19.8$ & $45.7 \pm 15.6$ & $<.001^{a}$ & .214 \\
\hline $\mathrm{LDL}$ (mg/dL) & $117.3 \pm 38.7$ & $118.2 \pm 34.7$ & $112.9 \pm 32.3$ & $85.4 \pm 32.7$ & $<.001^{a}$ & $<.001$ \\
\hline Triglycerides (mg/dL) & $95.5[78.5-113.7]$ & $\begin{array}{l}138.0 \\
{[104.0-182.7]}\end{array}$ & $124.5[88.0-165.5]$ & 94.0 [73.0-120.0] & $<.001^{d}$ & $<.001$ \\
\hline $\operatorname{ALT}(U I / I)^{c}$ & $17.0[13.0-22.0]$ & $25.0[18.0-37.0]$ & $55.0[41.5-104.5]$ & $32.0[22.0-43.0]$ & $<.001^{d}$ & .090 \\
\hline AST $(U \mathrm{II} /)^{\mathrm{c}}$ & 17.0 [16.0-21.0] & 20.5 [17.0-26.0] & 35 [27.0-51.5] & $38[25.2-55.2]$ & $<.001^{d}$ & $<.001$ \\
\hline GGT (UI/I) & $17.0[12.0-25.5]$ & $25.0[16.0-37.0]$ & $38.0[28.0-86.0]$ & 85.5 [38.0-173.0] & $<.001^{d}$ & $>.001$ \\
\hline Platelets $\left(\times 10^{9} / \mathrm{L}\right)$ & $230.6 \pm 57.7$ & $239.4 \pm 60.5$ & $228.6 \pm 54.4$ & $107.7 \pm 55.5$ & $<.001^{\mathrm{b}}$ & $<.001$ \\
\hline Albumin (g/dL) & $4.5 \pm 0.4$ & $4.4 \pm 0.3$ & $4.4 \pm 0.3$ & $3.5 \pm 0.7$ & $<.001^{\mathrm{b}}$ & $<.001$ \\
\hline Serum creatinine $(\mathrm{mg} / \mathrm{dL})^{c}$ & $0.9[0.7-0.9]$ & $0.9[0.7-1.0]$ & $0.9[0.8-1.0]$ & $0.9[0.7-1.2]$ & $.492^{d}$ & .432 \\
\hline LAL activity (nmol/spot/h) ${ }^{c}$ & $1.0[0.7-1.2]$ & $0.9[0.7-1.2]$ & $0.8[0.6-1.0]$ & $0.5[0.4-0.6]$ & $<.001^{d}$ & $<.001$ \\
\hline Statin (\%) & 47.7 & 43.2 & 21.7 & 7.0 & $<.001^{c}$ & $<.001$ \\
\hline
\end{tabular}

Abbreviations: ALT, alanine aminotransferase; AST, aspartate aminotransferase; CC, cryptogenic cirrhosis; GGT, $\gamma$-glutamil transferase; HDL, highdensity lipoprotein; LAL, lysosomal acid lipase; LDL, low-density lipoprotein; MACCE, major adverse cardiovascular and cerebrovascular events; NAFL, non-alcoholic fatty liver (ie 'simple steatosis'); NASH, non-alcoholic steatohepatitis.

aNOVA test.

${ }^{\mathrm{b}} \chi^{2}$ test.

${ }^{\mathrm{C}}$ Data reported as median and interquartile range.

${ }^{\mathrm{d}}$ Kruskal-Wallis test. 
FIGURE 2 Percentage of $\mathrm{LAL}<0.8 \mathrm{nmol} / \mathrm{spot} / \mathrm{h}$ across liver disease groups

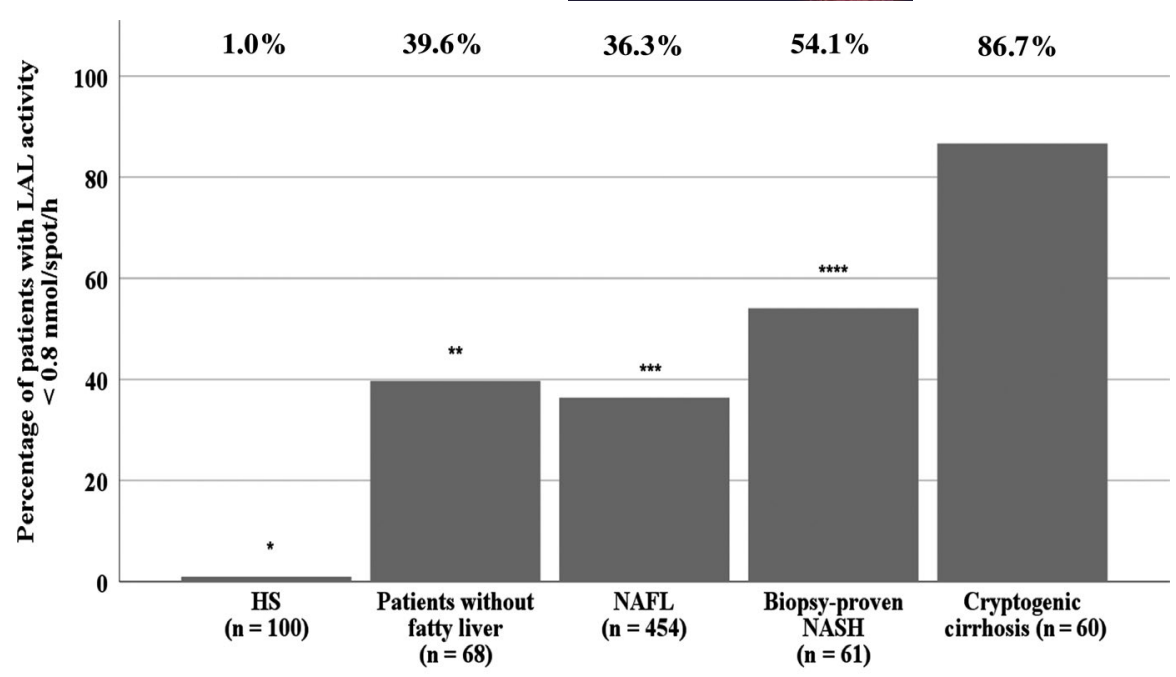

*HS vs patients without fatty liver $\mathrm{p}<0.001$, HS vs NAFL $\mathrm{p}<0.001$, HS vs NASH $\mathrm{p}<0.001$, HS vs CC p<0.001; **patients wihout fatty liver vs CC p<0.001; ***NAFL vs NASH $p=0.011$, NAFL vs CC p<0.001; ****NASH vs CC p<0.001 as compared to $4.6 \%$ in the intermediate and $0.5 \%$ in the highest tertile (Figure 3).

In Table 2, the multivariate logistic regression analysis showed that age $(O R=1.09, P<.001)$, diabetes $(O R=9.24, P=.006)$, $\log (A S T)(O R=7.74, P=.003)$, thrombocytopenia $(O R=16.03$, $<0.001), \log (L A L)(O R=0.12, P=.010)$ and statin use $(O R=0.13$, $P=.019)$ were independently associated with CC.

\subsection{Determinants of liver fibrosis in patients with NAFLD}

In NAFLD patients, $69.9 \%$ had a FIB4 < 1.30 , and $43.1 \%$ a NFS $<-1.455$. Patients with negative FIB-4 were younger and less likely to have arterial hypertension and previous MACCE than patients with FIB4 $\geq 1.30$ (Table 3, panel A). They also had higher LDL cholesterol and platelets and lower AST and GGT values. LAL activity was higher in patients with negative FIB-4 (Table 3, panel A). Similar results were obtained using NFS (Table 3, panel B).

Multivariate logistic regression analysis showed that log (LAL activity) and previous MACCE were associated with FIB-4 $<1.30$ (Table 4, model A). Using NFS as a dependent variable, we found that female sex, arterial hypertension, log (LAL activity) and previous MACCE were associated with NFS $<-1.455$ (Table 4, model B).

\section{3 | LAL activity and white blood cells}

In a subgroup of 330 NAFLD patients, we investigated the relationship between LAL activity and white blood cells count (WBC). We found a mean value of WBC of $6.65 \pm 1.77 \times 10^{3} / \mu \mathrm{l} ; \mathrm{WBC}$ and $\mathrm{LAL}$ activity were weakly correlated $(r S=0.215, P<.001)$. After adjustment for WBC, log (LAL activity)/log (WBC) remained significantly associated with Fib4 < $1.30(\mathrm{OR}=4.74 ; 95 \% \mathrm{Cl} 1.69-13.35, P=.003)$ and with NFS <-1.455 (OR $=4.20 ; 95 \% \mathrm{Cl} 1.61-10.96, P=.003)$ at univariable logistic regression analysis.

\section{4 | DISCUSSION}

Our cross-sectional study confirmed a progressive reduction in LAL activity with the severity of NAFLD, and we found a correlation between LAL activity and two validated non-invasive markers of liver fibrosis, namely FIB-4 and NFS.
FIGURE 3 Proportion of liver diseases across tertiles of LAL activity

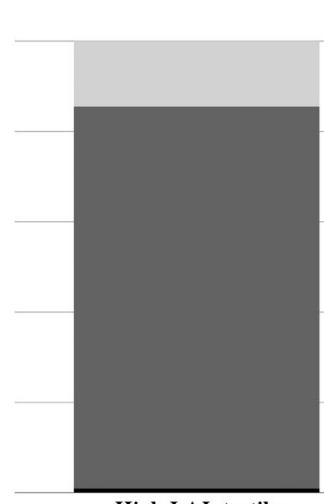

High LAL tertile

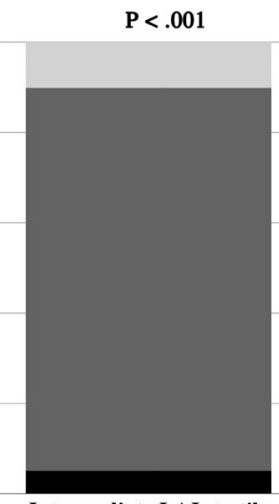

Intermediate LAL tertile

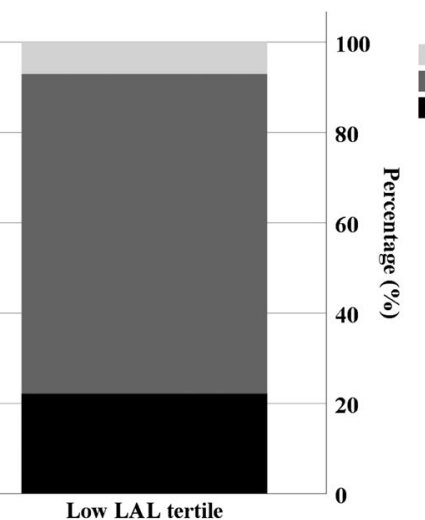

Low LAL tertile
No fatty liver NAFLD

Cirrhosis 


\begin{tabular}{|c|c|c|c|c|}
\hline & $\begin{array}{l}\text { Univariate analysis } \\
\text { OR }(95 \% \mathrm{Cl})\end{array}$ & $P$ & $\begin{array}{l}\text { Multivariate } \\
\text { analysis } \\
\text { OR }(95 \% \mathrm{CI})\end{array}$ & $P$ \\
\hline Age & $1.11(1.07-1.14)$ & $<.001$ & $1.09(1.02-1.17)$ & .011 \\
\hline Female sex & $0.57(0.32-1.02)$ & .058 & $0.28(0.06-1.44)$ & .129 \\
\hline Diabetes & $3.68(2.13-6.35)$ & $<.001$ & $9.24(1.90-44.9)$ & .006 \\
\hline Arterial hypertension & $0.41(0.24-0.71)$ & .001 & $0.29(0.06-1.38)$ & .117 \\
\hline Total cholesterol & $0.97(0.96-0.98)$ & $<.001$ & $0.99(0.97-1.00)$ & .114 \\
\hline $\log (\mathrm{AST})$ & $6.94(4.06-11.85)$ & $<.001$ & $7.74(2.05-29.17)$ & .003 \\
\hline $\begin{array}{l}\text { Thrombocytopenia } \\
\text { (platelets }<150 \times 10^{9} / \text { I) }\end{array}$ & $59.34(29.10-121.04)$ & $<.001$ & $16.03(3.68-82.62)$ & $<.001$ \\
\hline $\begin{array}{l}\text { Hypoalbumineamia } \\
\text { (albumin }<3.5 \mathrm{~g} / \mathrm{dL} \text { ) }\end{array}$ & $328.70(43.00-2512.49)$ & $<.001$ & $\begin{array}{l}24.88 \\
(0.84-739.53)\end{array}$ & .063 \\
\hline Log (LAL activity) & $0.03(0.01-0.06)$ & $<.001$ & $0.12(0.02-0.60)$ & .010 \\
\hline Statin use & $0.11(0.04-0.30)$ & $<.001$ & $0.13(0.02-0.71)$ & .019 \\
\hline
\end{tabular}

TAB LE 2 Univariate and multivariate logistic regression analysis of factors associated with cryptogenic cirrhosis

Abbreviations: AST, aspartate aminotransferase; $\mathrm{Cl}$, confidence interval; LAL, lysosomal acid lipase; OR, odds ratio.

TA B LE 3 Characteristics of 515 NAFLD patients with and without liver fibrosis according to FIB-4 (Panel A) and NFS (Panel B) scores

\begin{tabular}{|c|c|c|c|c|c|c|}
\hline & \multicolumn{3}{|l|}{ Panel A } & \multicolumn{3}{|l|}{ Panel B } \\
\hline Age (y) & $50.7 \pm 12.2$ & $60.7 \pm 9.4$ & $<.001$ & $50.7 \pm 12.2$ & $60.7 \pm 9.4$ & $<.001$ \\
\hline Women (\%) & 40.3 & 39.7 & .921 & 35.3 & 42.6 & .101 \\
\hline Diabetes (\%) & 28.0 & 34.5 & .489 & 13.3 & 42.6 & $<.001$ \\
\hline Previous MACCE (\%) & 3.2 & 11.5 & .001 & 0.5 & 9.3 & $<.001$ \\
\hline Blood glucose (mg/dL) & $104.8 \pm 27.9$ & $105.6 .4 \pm 19.5$ & .753 & $96.0 \pm 18.1$ & $112.4 \pm 28.6$ & $<.001$ \\
\hline Total cholesterol (mg/dL) & $199.3 \pm 38.1$ & $192.2 \pm 41.7$ & .065 & $203.8 \pm 40.0$ & $192.6 \pm 38.3$ & .001 \\
\hline $\mathrm{HDL}(\mathrm{mg} / \mathrm{dL})$ & $47.8 \pm 12.9$ & $50.2 \pm 16.1$ & .074 & $49.1 \pm 15.8$ & $48.0 \pm 12.3$ & .399 \\
\hline AST (UI/I) & $20.0[17.0-26.0]$ & $25.0[20.0-39.5]$ & $<.001$ & 22 [18.0-28.0] & 21 [17.0-29.0] & .361 \\
\hline GGT (UI/I) & $25.0[17.0-37.0]$ & $30.0[17.5-48.5]$ & .027 & $27.0[17.0-43.7]$ & $26.0[17.0-38.0]$ & .437 \\
\hline Platelets $\left(\times 10^{9} / \mathrm{I}\right)$ & $256.8 \pm 56.8$ & $194.5 \pm 42.1$ & $<.001$ & $269.0 \pm 58.5$ & $214.1 \pm 48.8$ & $<.001$ \\
\hline Albumin (g/l) & $4.4 \pm 0.4$ & $4.4 \pm 0.3$ & .363 & $4.5 \pm 0.4$ & $4.3 \pm 0.3$ & $<.001$ \\
\hline Serum creatinine $(\mathrm{mg} / \mathrm{dL})$ & $0.9[0.7-0.9]$ & $0.9[0.7-1.1]$ & .110 & $0.9[0.8-1.0]$ & $0.9[0.7-1.0]$ & .712 \\
\hline LAL activity (nmol/spot/h) & $0.9[0.7-1.2]$ & $0.8[0.6-1.0]$ & .008 & $0.9[0.7-1.2]$ & $0.8[0.6-1.1]$ & .001 \\
\hline Statin use (\%) & 38.8 & 48.3 & .069 & 33.5 & 46.6 & .04 \\
\hline
\end{tabular}

Note: See Table 1 for abbreviation list.

These findings confirm the results from previous studies showing a significant reduction of LAL activity in NAFLD patients, compared to $\mathrm{HS}$, with a further reduction in the subgroup of those with $\mathrm{NASH}^{4,5}$ In particular, patients with NAFL had a $27.0 \%$ reduction in LAL activity, which increased to $41.8 \%$ in the subgroup of patients with biopsy-proven NASH.
Despite we found a significant reduction of LAL activity in patients with NAFLD and CC, this was not compatible with a genetic deficiency of $L A L$ in which the residual enzimatic activity is usually $<15 \%$.

A new finding of the present study is the significant inverse association between LAL activity and liver fibrosis assessed 
TAB LE 4 Multivariate logistic regression analysis of factors associated with FIB-4 $<1.30$ (model A) and NFS < -1.455 (model $B$ ), in 515 patients with NAFLD

\begin{tabular}{|c|c|c|c|c|}
\hline & \multicolumn{2}{|l|}{ Model A } & \multicolumn{2}{|l|}{ Model B } \\
\hline & FIB- $4<1.30$ & & NFS $<-1.455$ & \\
\hline & OR $[95 \% \mathrm{Cl}]$ & $P$ & OR $[95 \% \mathrm{Cl}]$ & $P$ \\
\hline Female sex & $0.94(0.62-1.45)$ & .794 & $0.67(0.45-0.98)$ & .040 \\
\hline Diabetes & $0.94(0.59-1.50)$ & .805 & - & \\
\hline Arterial hypertension & $0.78(0.50-1.22)$ & .273 & $0.47(0.32-0.69)$ & $<.001$ \\
\hline LDL cholesterol & $1.00(1.00-1.01)$ & .136 & $1.00(1.00-1.01)$ & .216 \\
\hline Log (LAL activity) & $2.19(1.33-3.62)$ & .002 & $2.43(1.51-3.91)$ & $<.001$ \\
\hline $\begin{array}{l}\text { High waist } \\
\text { circumference }\end{array}$ & $0.85(0.49-1.47)$ & .562 & - & \\
\hline Previous MACCE & $0.34(0.15-0.77)$ & .010 & $0.06(0.01-0.49)$ & .008 \\
\hline
\end{tabular}

Abbreviations: $\mathrm{Cl}$, confidence interval; LAL, lysosomal acid lipase; LDL, low density lipoprotein; MACCE, major advanced cardiovascular and cerebrovascular events; OR, odds ratio. by FIB-4 score and NFS indexes, two well-validated surrogate markers of fibrosis. In particular, the FIB-4 index has been reported to be superior to seven other non-invasive markers of fibrosis in patients with NAFLD to identify a high probability of severe fibrosis. ${ }^{20}$ Moreover, it has the advantage of having been validated for both HCV and NAFLD, two common chronic liver diseases.

To corroborate our results, we repeated our analysis using another validated index of fibrosis, the NFS, which is a more accurate scoring system, based on six routinely measured parameters, which stratifies fibrosis along the wide continuum of NAFLD. These findings are in agreement with Shteyer et $\mathrm{al}^{24}$ who found that low LAL activity correlates with advanced liver disease and that LAL activity $<0.5$ indicates severe liver injury in patients with fatty liver and cirrhosis. Finally, they are also in keeping with Selvakumar PK et al who reported a correlation between reduced blood LAL activity and the severity of liver fibrosis in children with NAFLD. ${ }^{8}$

Recently, the clinical outcomes of 18 patients undergoing liver transplantation with LAL deficiency have been reported. The study demonstrates that liver transplantation may be necessary for LAL-D-associated liver failure, but is neither sufficient to increase LAL activity, nor to prevent disease progression, or liver disease recurrence. ${ }^{25}$ This is probably because of the fact that the pathophysiology of LAL-D is predominantly mediated by deficient enzyme activity in bone marrow-derived monocyte-macrophages.

In a Phase 3 interventional clinical trial, performed in LAL-D patients, sebelipase supplementation reduced the hepatic fat content compared with baseline $(-32 \%, P=.001$ vs placebo), and also caused a normalization of ALT and AST values, suggesting therefore a potential effect on reducing the risk of fibrosis. ${ }^{26}$ Furthermore, sebelipase alfa supplementation seems to have a positive impact on surrogate markers of atherosclerosis, ${ }^{27}$ a beneficial 'pleiotropic' effect which may turn useful in patients with NAFLD who experience a high rate of cardiovascular complications. ${ }^{28,29}$ Moreover, in experimental models, supplementation of $L A L$ resulted in the regression of coronary and aortic atheromatous lesions. ${ }^{30}$ This could be of greater interest to patients with the more advanced liver disease, such as those with NASH or CC, for whom we do not currently have effective therapies.

Another interesting finding of our study relates to the use of statins. Thus, statin use was inversely associated with cirrhosis, confirming a potential efficacy and safety of statins in patients with NAFLD, ${ }^{31}$ and their potential effectiveness in reducing progression to cirrhosis. ${ }^{32,33}$

The study has implications and limitations. Strength of the study is the very large series of patients studied across the wide clinical continuum of NAFLD from NAFL to CC. However, one major limitation is that it is a single-centre cross-sectional study, and we, therefore, cannot establish a cause-effect relationship between the reduction in LAL activity and the presence of liver damage. Thus, until new data become available, the reduction in LAL activity may be regarded to only as a marker of NAFLD severity. ${ }^{34}$ Furthermore, liver fibrosis was estimated by non-invasive biomarkers, that despite being well validated, represent an indirect measure and do not provide neither a quantitative or qualitative evaluation of liver fibrosis.

In conclusion, our study showed a significant association between LAL activity and non-invasive markers of liver fibrosis. Longitudinal studies are needed to establish an association between $\mathrm{LAL}$ activity reduction and risk of fibrosis.

\section{ACKNOWLEDGEMENTS}

We thank Nurse Daniela Salzano for her skilful collaboration. The Whatman grade 903 papers to assess LAL activity were provided by an unrestricted liberal donation from Alexion Pharmaceuticals, Inc, which has no access to any clinical or biochemical data.

\section{ORCID}

Daniele Pastori (iD https://orcid.org/0000-0001-6357-5213

Francesco Violi (iD https://orcid.org/0000-0002-6610-7068

Francesco Angelico (iD https://orcid.org/0000-0002-9372-3923 


\section{REFERENCES}

1. Angelico F, Del Ben M, Conti R, et al. Insulin resistance, the metabolic syndrome, and nonalcoholic fatty liver disease. J Clin Endocrinol Metab. 2005;90(3):1578-1582.

2. Baratta F, Pastori D, Polimeni L, et al. Adherence to mediterranean diet and non-alcoholic fatty liver disease: effect on insulin resistance. Am J Gastroenterol. 2017;112(12):1832-1839.

3. Angulo P, Kleiner DE, Dam-Larsen S, et al. Fibrosis, but no other histologic features, is associated with long-term outcomes of patients with nonalcoholic fatty liver disease. Gastroenterology. 2015;149(2):389-397.e10.

4. Baratta F, Pastori D, Del Ben M, et al. Reduced lysosomal acid lipase activity in adult patients with non-alcoholic fatty liver disease. EBioMedicine. 2015;2(7):750-754.

5. Tovoli F, Napoli L, Negrini G, et al. A relative deficiency of lysosomal acid lypase activity characterizes non-alcoholic fatty liver disease. Int J Mol Sci. 2017;18(6):1134.

6. Angelico F, Corradini SG, Pastori D, et al. Severe reduction of blood lysosomal acid lipase activity in cryptogenic cirrhosis: a nationwide multicentre cohort study. Atherosclerosis. 2017;262:179-184.

7. Vespasiani-Gentilucci U, Gallo P, Piemonte F, et al. Lysosomal acid lipase activity is reduced both in cryptogenic cirrhosis and in cirrhosis of known etiology. PLoS ONE. 2016;11(5):e0156113.

8. Selvakumar PK, Kabbany MN, Lopez R, et al. Reduced lysosomal acid lipase activity - A potential role in the pathogenesis of non alcoholic fatty liver disease in pediatric patients. Dig Liver Dis. 2016;48:909-913.

9. Grundy SM, Cleeman JI, Daniels SR, et al. Diagnosis and management of the metabolic syndrome: an American Heart Association/ National Heart, Lung, and Blood Institute Scientific Statement. Circulation. 2005;112(17):2735-2752.

10. Authors/Task Force Members, Ryden L, Grant PJ, et al. ESC Guidelines on diabetes, pre-diabetes, and cardiovascular diseases developed in collaboration with the EASD: The Task Force on diabetes, pre-diabetes, and cardiovascular diseases of the European Society of Cardiology (ESC) and developed in collaboration with the European Association for the Study of Diabetes (EASD). Eur Heart J. 2013;34(39):3035-3087.

11. Mancia G, Fagard R, Narkiewicz K, et al. 2013 Practice guidelines for the management of arterial hypertension of the European Society of Hypertension (ESH) and the European Society of Cardiology (ESC): ESH/ESC Task Force for the Management of Arterial Hypertension. J Hypertens. 2013;31(10):1925-1938.

12. Hamaguchi M, Kojima T, Itoh $\mathrm{Y}$, et al. The severity of ultrasonographic findings in nonalcoholic fatty liver disease reflects the metabolic syndrome and visceral fat accumulation. Am J Gastroenterol. 2007;102(12):2708-2715.

13. Bush K, Kivlahan DR, Mcdonell MB, Finn SD, Bradley KA. The AUDIT alcohol consumption questions (AUDIT-C): an effective brief screening test for problem drinking. Ambulatory Care Quality Improvement Project (ACQUIP). Alcohol Use Disorders Identification Test. Arch Intern Med. 1998;158(16):1789-1795.

14. Kim MY, Jeong WK, Baik SK. Invasive and non-invasive diagnosis of cirrhosis and portal hypertension. World J Gastroenterol. 2014;20(15):4300-4315.

15. Goyal N, Jain N, Rachapalli V, Cochlin DL, Robinson M. Non-invasive evaluation of liver cirrhosis using ultrasound. Clin Radiol. 2009;64(11):1056-1066.

16. Kleiner DE, Brunt EM, Van Natta M, et al. Design and validation of a histological scoring system for nonalcoholic fatty liver disease. Hepatology. 2005;41(6):1313-1321.

17. Carpino G, Pastori D, Baratta F, et al. PNPLA3 variant and portal/periportal histological pattern in patients with biopsy-proven non-alcoholic fatty liver disease: a possible role for oxidative stress. Sci Rep. 2017;7(1):15756.

18. Caldwell SH, Oelsner DH, lezzoni JC, Hespenheide EE, Battle EH, Driscoll CJ. Cryptogenic cirrhosis: clinical characterization and risk factors for underlying disease. Hepatology. 1999;29(3):664-669.

19. Shah AG, Lydecker A, Murray K, Tetri BN, Contos MJ, Sanyal AJ. Comparison of noninvasive markers of fibrosis in patients with nonalcoholicfattyliverdisease. Clin Gastroenterol Hepatol.2009;7(10):1104-1112.

20. McPherson S, Stewart SF, Henderson E, Burt AD, Day CP. Simple non-invasive fibrosis scoring systems can reliably exclude advanced fibrosis in patients with non-alcoholic fatty liver disease. Gut. 2010;59(9):1265-1269.

21. Unalp-Arida A, Ruhl CE. Liver fibrosis scores predict liver disease mortality in the United States population. Hepatology. 2017;66(1):84-95.

22. Di Costanzo A, Belardinilli F, Bailetti D, et al. Evaluation of polygenic determinants of non-alcoholic fatty liver disease (NAFLD) by a candidate genes resequencing strategy. Sci Rep. 2018;8(1):3702.

23. Minicocci I, Pozzessere S, Prisco C, et al. Analysis of children and adolescents with familial hypercholesterolemia. J Pediatr. 2017;183:100-107.e3.

24. Shteyer E, Villenchik R, Mahamid M, Nator N, Safadi R. Low serum lysosomal acid lipase activity correlates with advanced liver disease. Int J Mol Sci. 2016;17(3):312.

25. Bernstein DL, Lobritto $S$, luga $A$, et al. Lysosomal acid lipase deficiency allograft recurrence and liver failure- clinical outcomes of 18 liver transplantation patients. Mol Genet Metab. 2018;124(1):11-19.

26. Burton BK, Balwani M, Feillet $F$, et al. A phase 3 trial of sebelipase alfa in Iysosomal acid lipase deficiency. N EnglJ Med. 2015;373(11):1010-1020.

27. Wilson DP, Friedman M, Marulkar S, Hamby T, Bruckert E. Sebelipase alfa improves atherogenic biomarkers in adults and children with lysosomal acid lipase deficiency. J Clin Lipidol. 2018;12(3):604-614.

28. Pastori D, Baratta F, Novo M, et al. Lipoprotein cholesterol and cardiovascular and cerebrovascular events in patients with non-alcoholic fatty liver disease. J Clin Med. 2018;7(11):378.

29. del Ben M, Baratta F, Polimeni L, Angelico F. Non-alcoholic fatty liver disease and cardiovascular disease: epidemiological, clinical and pathophysiological evidences. Intern Emerg Med. 2012;7(Suppl 3):S291-S296.

30. Du H, Schiavi S, Wan N, Levine M, Witte DP, Grabowski GA Reduction of atherosclerotic plaques by lysosomal acid lipase supplementation. Arterioscler Thromb Vasc Biol. 2004;24(1):147-154.

31. Pastori D, Polimeni L, Baratta F, Pani A, del Ben M, Angelico F. The efficacy and safety of statins for the treatment of non-alcoholic fatty liver disease. Dig Liver Dis. 2015;47(1):4-11.

32. Imprialos KP, Stavropoulos K, Doumas M, Skalkou A, Zografou I, Athyros VG. The potential role of statins in treating liver disease. Expert Rev Gastroenterol Hepatol. 2018;12(4):331-339.

33. Kamal S, Khan MA, Seth A, et al. Beneficial effects of statins on the rates of hepatic fibrosis, hepatic decompensation, and mortality in chronic liver disease: a systematic review and meta-analysis. Am J Gastroenterol. 2017;112(10):1495-1505.

34. Cunha-Silva M, Mazo D, Corrêa BR, et al. Lysosomal acid lipase deficiency leading to liver cirrhosis: a case report of a rare variant mutation. Ann Hepatol. 2018;18(1):78-88.

How to cite this article: Baratta F, Pastori D, Tozzi G, et al. Lysosomal acid lipase activity and liver fibrosis in the clinical continuum of non-alcoholic fatty liver disease. Liver Int. 2019;00:1-8. https://doi.org/10.1111/liv.14206 